\title{
Three-dimensional reconnection on the Sun
}

\author{
Eric R. Priest \\ Department of Mathematics and Statistics, University of St Andrews, St Andrews, Fife, KY16 9SS, U.K.
}

(Received May 1, 2000; Revised November 1, 2000; Accepted November 1, 2000)

\begin{abstract}
A brief review is given of the theory of magnetic reconnection in three dimensions. The key elements of a threedimensional null point are its spine and its fan, which consist, respectively, of a field line and a surface of field lines that pass through the null. The fans of two nulls intersect in general in a field line called a separator that joins the nulls. Several different types of reconnection have been proposed, namely: spine reconnection, fan reconnection, separator reconnection and quasi-separatrix layer reconnection. In addition, a new exact solution for reconnective annihilation has been recently discovered. A summary is also given of the impressive evidence of reconnection at work on the Sun provided by a range of observations from the Yohkoh and SOHO satellites, both of solar flares and of coronal heating events.
\end{abstract}

\section{Introduction}

Two-dimensional magnetohydrodynamic reconnection is now fairly well understood. The classical Sweet-Parker and Petschek regimes have been generalised to give an AlmostUniform family (Priest and Forbes, 1986) and a NonUniform family (Priest and Lee, 1990; Strachan and Priest, 1994) of regimes which depend partly on the boundary conditions and partly on whether the magnetic diffusivity is uniform or nonuniform. Recently, Terry Forbes and I have completed a monograph (Priest and Forbes, 2000) that describes these models in detail, as well as models for unsteady and three-dimensional reconnection and their application to the magnetosphere, the Sun, astrophysics, the laboratory and particle acceleration.

There are also few exact solutions of the MHD equations. For magnetic annihilation (Sonnerup and Priest, 1975) the magnetic flux function is

$$
A=A_{0}(x)
$$

and the stream function is

$$
\psi=v_{e x} x y
$$

and one can impose the values of the inflow speed $\left(v_{e x}\right)$ and inflow magnetic field $\left(B_{y e}\right)$ at an inflow point $(x, y)=$ $(1,0)$, say. More recently, Craig and Henton (1995) suggested a solution

$$
\begin{aligned}
& A=A(x)+v_{x e} \frac{v_{y e}}{B_{y e}} x y \\
& \psi=x y+A(x)
\end{aligned}
$$

in which they were also able to impose $v_{y}=v_{y e}$ at $(x, y)=$ $(1,0)$. Recently, Priest et al. (2000a) have discovered much

Copy right (c) The Society of Geomagnetism and Earth, Planetary and Space Sciences (SGEPSS); The Seismological Society of Japan; The Volcanological Society of Japan; The Geodetic Society of Japan; The Japanese Society for Planetary Sciences. more general reconnective annihilation solutions of the form

$$
\begin{aligned}
& A=A_{0}(x)+A_{1}(x) y \\
& \psi=\psi_{0}(x)+\psi_{1}(x) y
\end{aligned}
$$

in which one can also impose $B_{x}$ at $(1,0)$ and there is an additional free parameter. Also, it is currently being extended into three dimensions.

In this brief review we first of all describe some of the attempts to extend ideas about two-dimensional reconnection into three dimensions and then we summarise some of the recent evidence for the presence of reconnection of work in the Sun's corona. We consider only reconnection at null points where the magnetic field vanishes, although there are other regimes that occur in the absence of null points (Schindler et al., 1988). Also, we focus on the MHD aspects of reconnection, which apply to collisional environments such as the solar photosphere. In regions such as the solar corona where the diffusion region is collisionless, the MHD models still play an important role in providing the macroscopic environment within which the microscopic collisionless processes are at work. Indeed, a proper linking of the MHD and collisionless aspects is an important matter for future research.

\section{Three-Dimensional Magnetic Reconnection}

When we go from two dimensions into three dimensions, there are many new features that are currently being studied (Sato, 1985; Hesse and Schindler, 1988; Lau and Finn, 1990; Shibata et al., 1996; Longcope and Cowley, 1996; Priest and Titov, 1996; Ugai and Wang, 1998). For example, the definition itself is no longer obvious. The structure of the null points is different. The global topology of the field is much more complex, and there are several different types of magnetic reconnection. In particular, it was Axford (1984) who stressed that a change of magnetic connectivity is the key to a definition of three-dimensional reconnection and Schindler et al. (1988) who proposed a notion of general magnetic reconnection. 


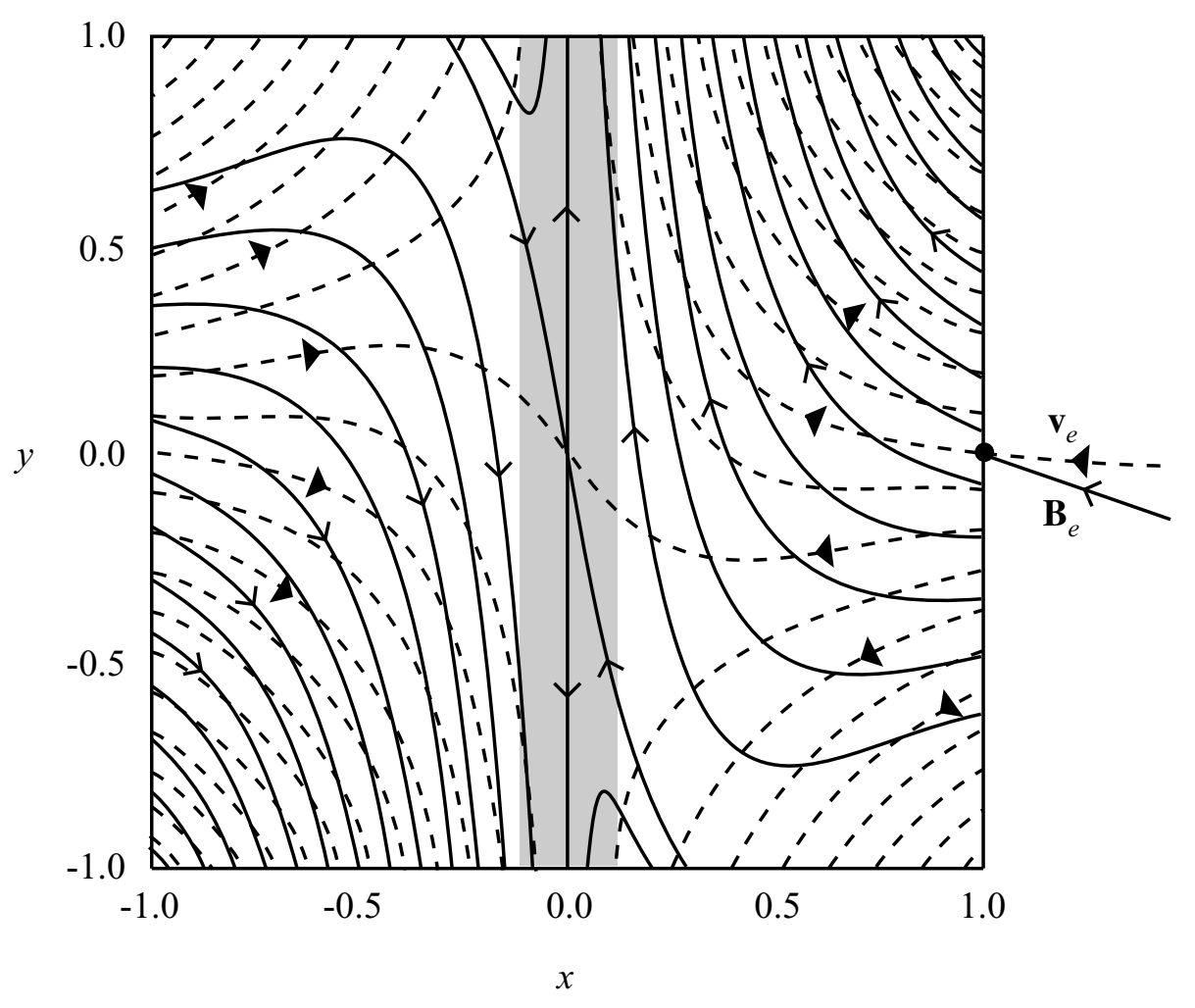

Fig. 1. Magnetic field lines and streamlines for reconnective annihilation (Priest et al., 2000a).

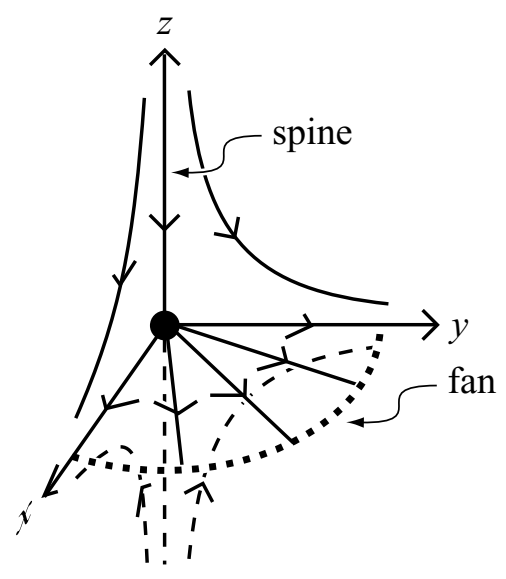

Fig. 2. Magnetic structure of a three-dimensional null point.

\subsection{Structure of a null point}

The simplest magnetic null in three dimensions has magnetic field components

$$
\left(B_{x}, B_{y}, B_{z}\right)=(x, y,-2 z)
$$

which satisfy $\nabla \cdot \mathbf{B}=0$. The resulting magnetic field lines have the structure shown in Fig. 2, in which there are two families of magnetic field lines through the null point. A spine approaches the origin from above and below along the $z$-axis. Also, a fan of field lines leaves the origin in a surface (the $x y$-plane). More generally, the field near a linear null always possesses a spine and a fan, but the field lines in the null may spiral or touch an axis and the spine may be

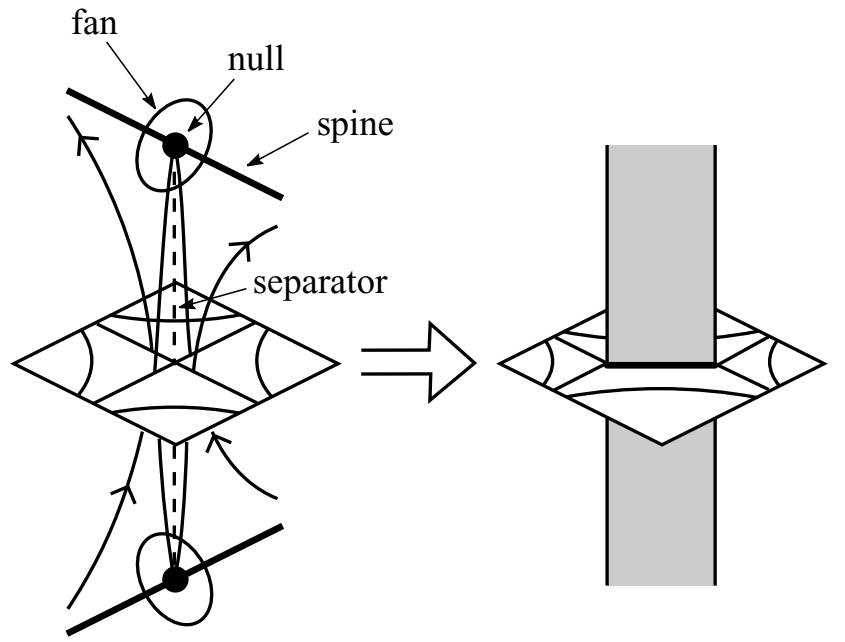

Fig. 3. Separator reconnection due to the collapse of a separator.

inclined to the fan at some other angle than $\frac{1}{2} \pi$ (Parnell et al., 1996).

\subsection{Types of reconnection}

At a magnetic null point, three different types of reconnection have been discovered. In spine reconnection the current is concentrated along the spine (Priest and Titov, 1996). It may be driven by continuous footpoint motions across the fan of a null point. In fan reconnection the current concentrates along the fan, and it may be driven by continuous footpoint motions across the spine of a null (Priest and Titov, 1996). The result is a rapid counter-flipping of magnetic field lines above and below the fan, which has been observed 


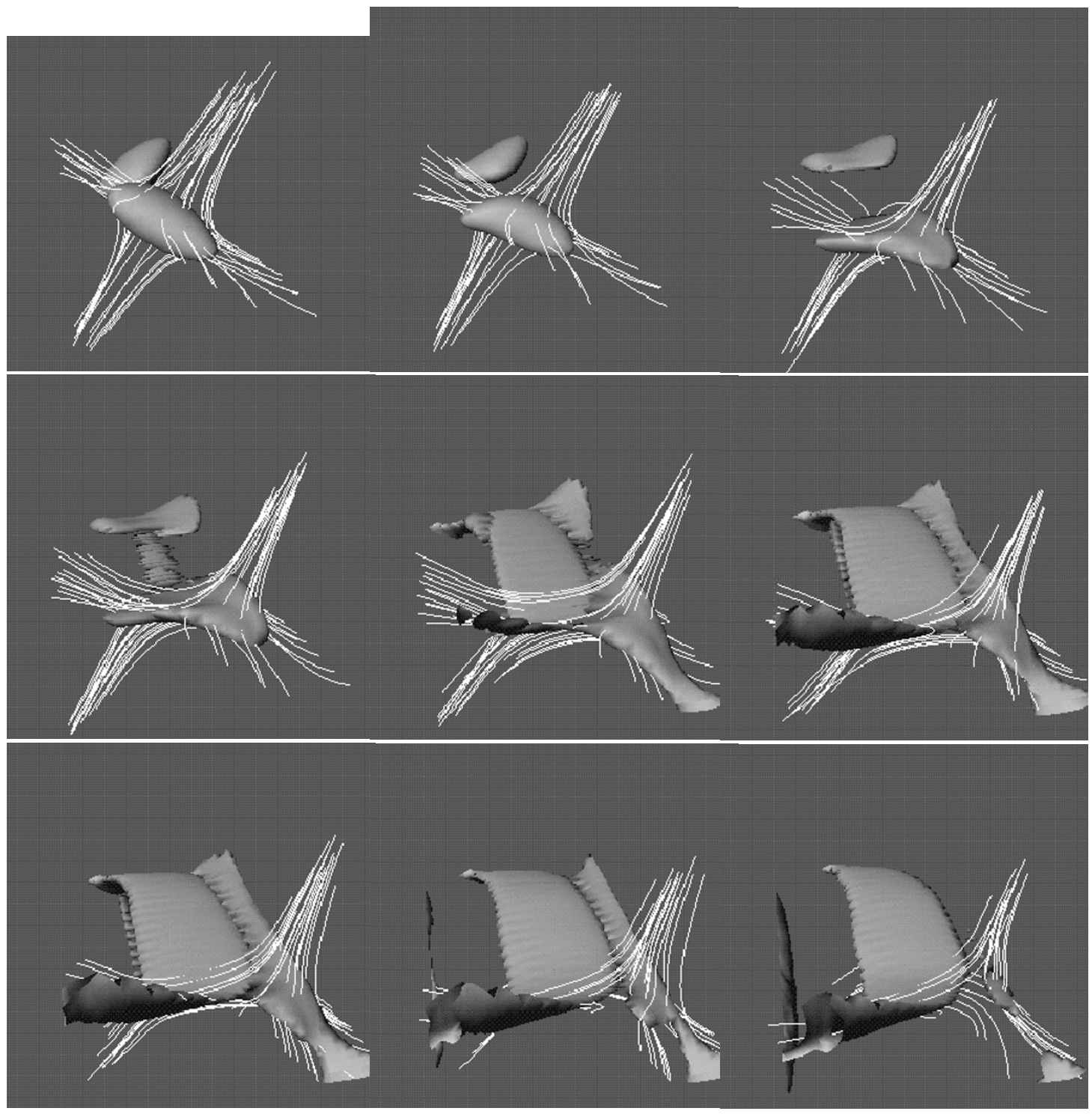

Fig. 4. Numerical experiment with an initial configuration of 8 null points, showing the local collapse of one of the nulls and the development of a strong current joining a nearby null due to separator reconnection (Galsgaard and Nordlund, 1997).

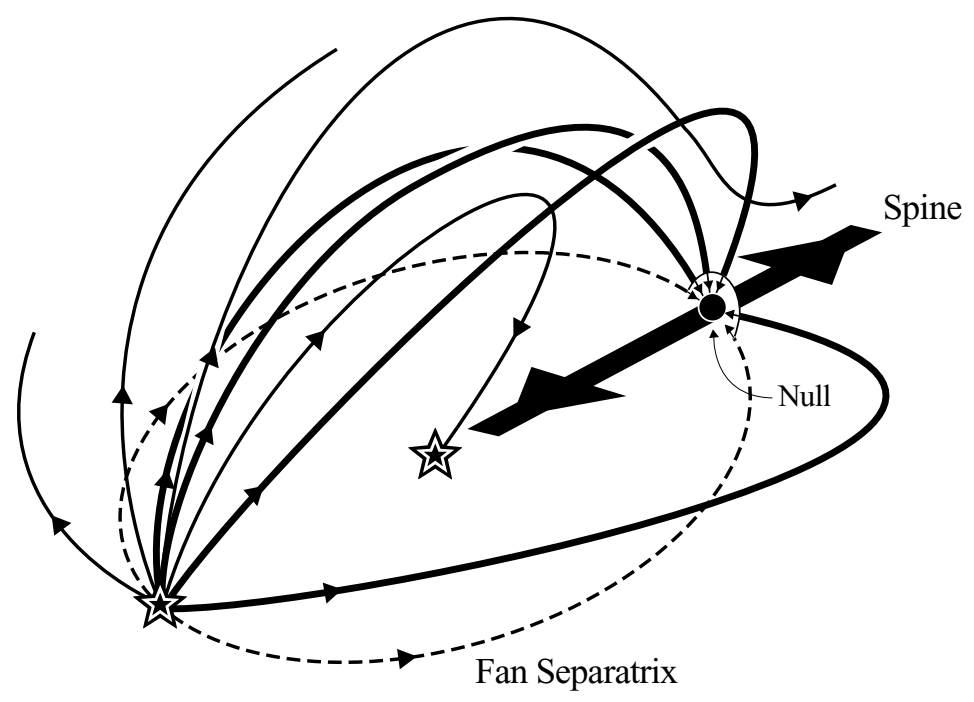

Fig. 5. The skeleton of two unbalanced sources (stars) in an ambient magnetic field, namely a null (a large dot) a spine (thick solid curve) and a separatrix fan that intersects the horizontal plane in a dashed curve. 
in the solar atmosphere by the TRACE satellite (Priest and Schrijver, 1999).

In separator reconnection (Fig. 3) the current is concentrated along a separator, which is the intersection of the fans of two nulls and so is the magnetic field line that joins one null to another (Priest and Titov, 1996; Longcope and Silva, 1998). Reconnection may also occur in the absence of nulls at so-called quasi-separatrix layers, where the gradient of the mapping from one boundary to another is very large (Priest and Demoulin, 1995; Hornig and Rastätter, 1998; Titov et al., 1999). Indeed, Demoulin et al. (1996) have demonstrated that noneruptive flares are often located along quasiseparatrix layers.

These different three-dimensional mechanisms have been analysed both in the kinematic approximation and in the strong magnetic field approximation of the full MHD equations and also numerically.

For example, Galsgaard and Nordlund (1997) started with a magnetic field consisting of eight null points in force-free equilibrium and then sheared the footpoints and watched what happened in a three-dimensional resistive MHD experiment. The nulls collapsed and reconnected by separator reconnection with strong current sheets joining nearby nulls (Fig. 4).

\subsection{Complex magnetic topology}

In two dimensions there are separatrix curves that separate the plane up into topologically distinct regions, and a pair of separatrices intersects in an X-type neutral point where the magnetic field vanishes. In three dimensions separatrix surfaces divide up the volume into topologically separate regions and separatrices intersect in a special field line known as a separator, which ends either at null points or on the boundary. In the Sun's surface there is a multitude of flux sources, and so the topology of the overlying coronal magnetic field is extremely complex. However, it can be described in terms of its skeleton, namely, a set of null points, a network of spine curves and a collection of separatrix fan surfaces. Its building blocks are the fields due to two, three and four sources. For two sources there is a null, with its

\section{Separatrix surface}

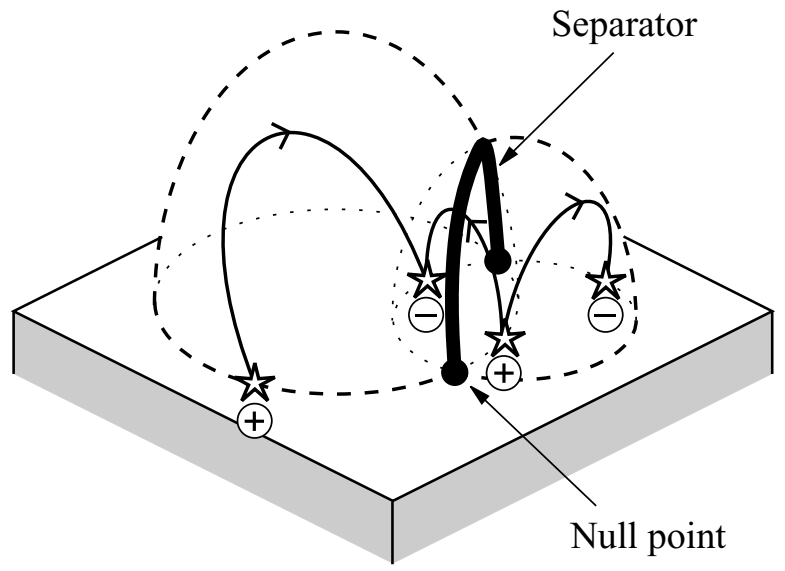

Fig. 6. The skeleton due to four sources showing two separatrix surfaces intersecting in a separator.

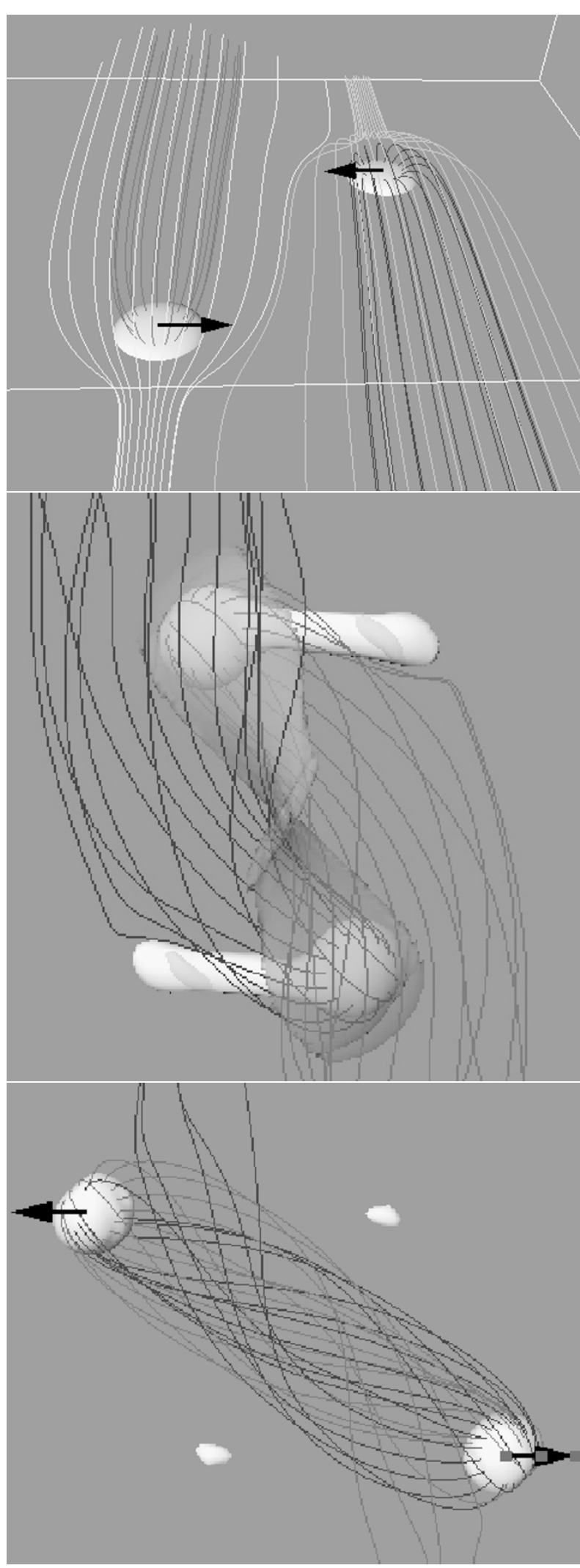

Fig. 7. Numerical experiment on separator reconnection, showing (a) the initial state, (b) the current sheet during reconnection and (c) the final state (Galsgaard et al., 2000). 


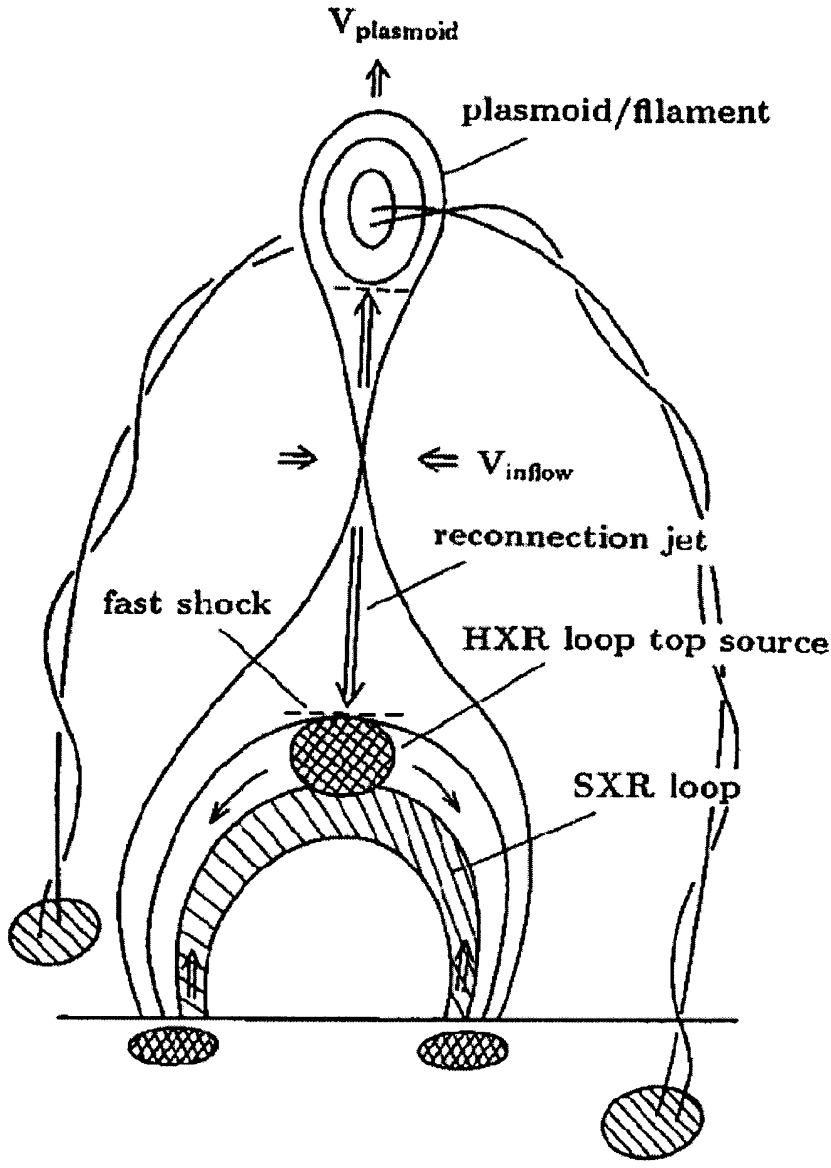

Fig. 8. Shibata's model of a solar flare. spine and separatrix surface that intersects the solar surface in the dashed curve shown in Fig. 5. For three sources the structure is surprisingly rich, with six different topological states when two sources are of the same sign and differ in sign from the third source. Transfer from one state to another is by local or global bifurcations (Priest et al., 1996).

So what is the effect on the corona of motions of magnetic

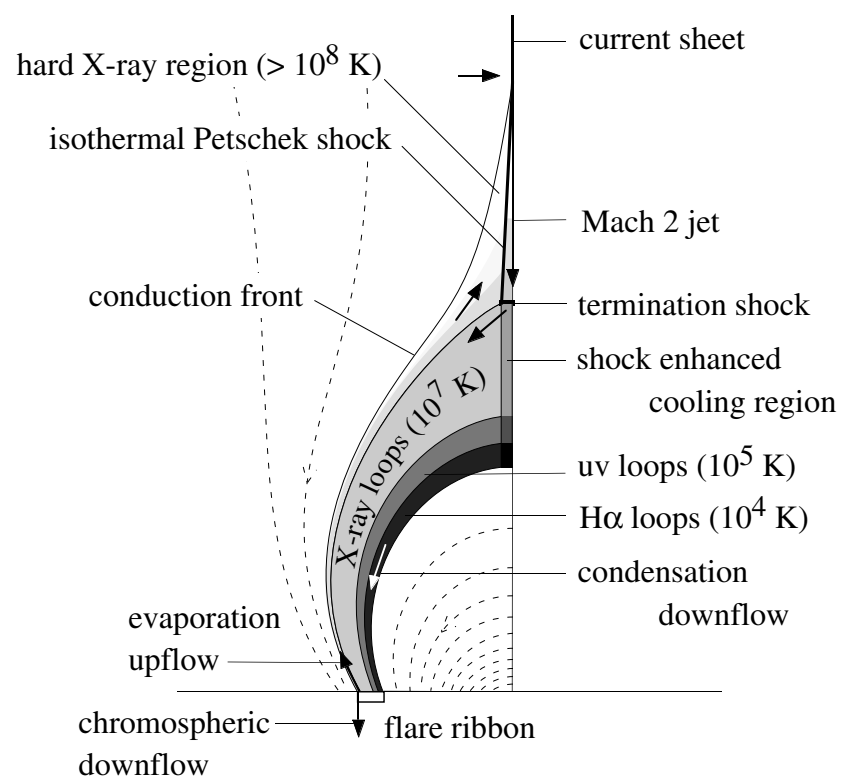

Fig. 9. The structure of flare loops created by reconnection (Priest and Forbes, 2000).

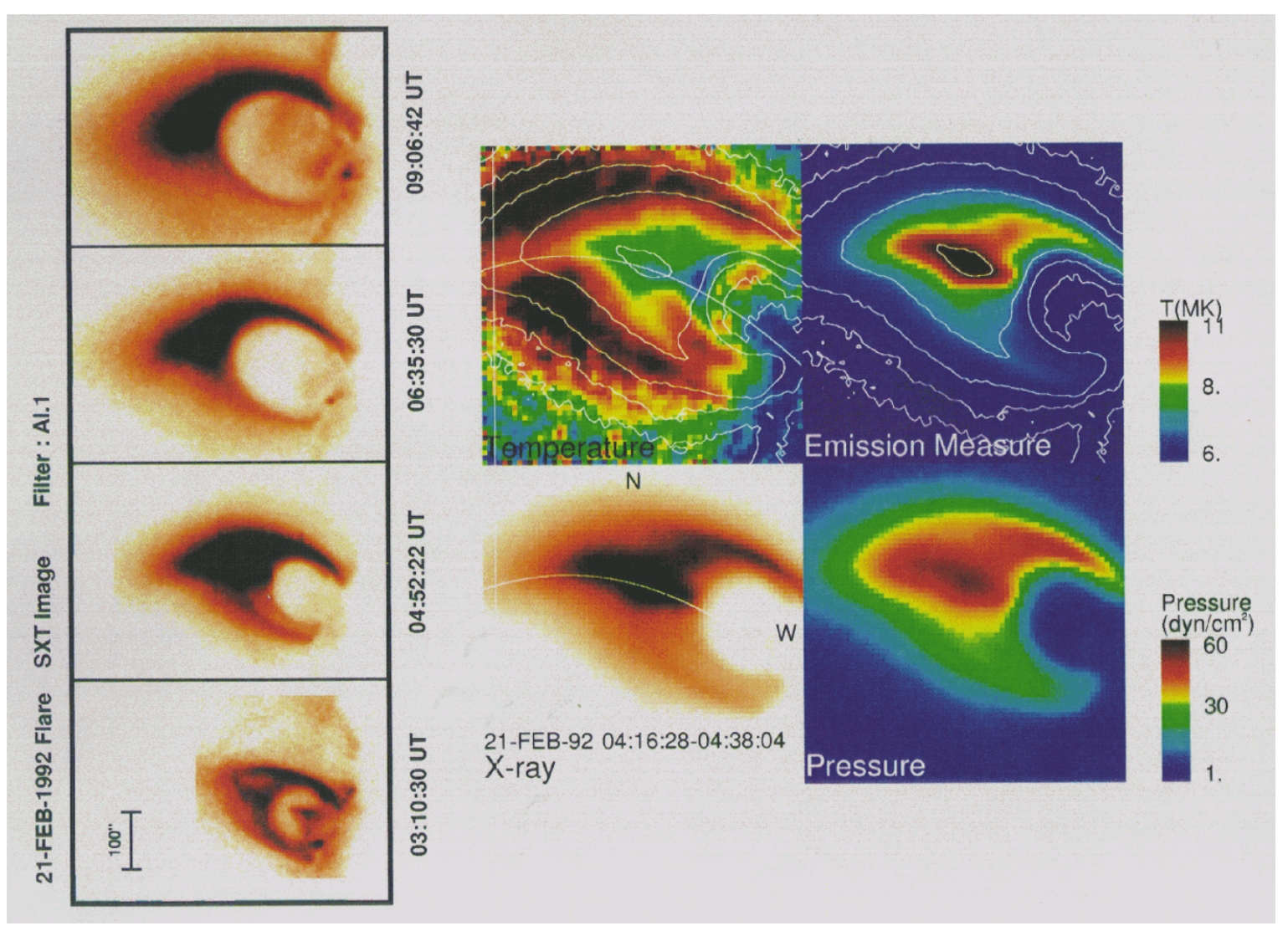

Fig. 10. The structure of the Tsuneta flare. 

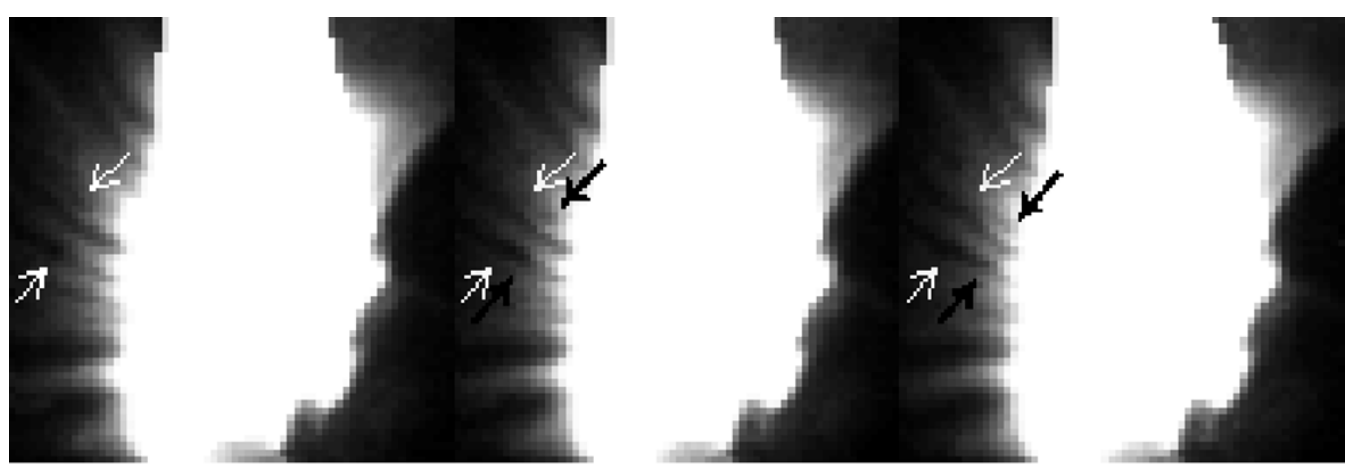

$21 \div 08 \div 49$

$21 \div 10 \div 57$

$21 \div 13 \div 05$

Fig. 11. Spikey current sheet above a flare loop system (McKenzie and Hudson, 1999).

flux sources in the solar surface? It drives three-dimensional reconnection and therefore heating. There are two main elements of this process, depending on whether the fundamental interaction is between a pair of sources or between three or more sources. First of all, the relative motion of a pair of sources in an ambient field (Fig. 5) produces heating by binary reconnection (Priest and Schrijver, 1999). Just as in a gas, the binary collisions between pairs of particles dominates, so in the corona the effect of the interactions of pairs of photospheric magnetic flux sources may dominate the resulting heating that is driven in the corona. As the sources move around, the flux joining them is preserved, but individual magnetic field lines are reconnected since they may either lie below the separatrix dome or above it, depending on the orientation of the sources. The resulting heating is focussed on the separatrix surface and along the field lines that have just crossed it. The second element is an effect of the interaction of three or four sources, namely, separator reconnection when their separatrix surfaces intersect (Priest and Titov, 1996; Longcope, 1998). As such sources move, there is now a change of flux in each region as flux is transferred from one to another, and the heat is concentrated along the separatrices and especially along the separator (Fig. 6).

Galsgaard et al. (2000) have recently set up a numerical experiment for a scenario proposed by Longcope (1998) for the interaction between two flux sources in an ambient field. Initially, the sources are not connected magnetically (Fig. 7(a)). As they move, separator reconnection occurs and produces a twisted current sheet along the separator (Fig. 7(b)). After reconnection has occurred, the two sources are joined by a twisted flux tube, which has been created by the conversion of mutual magnetic helicity in the initial state to the self-helicity of the flux tube.

\section{Recent Observational Evidence for Reconnec- tion}

There is now a host of indirect evidence in favour of reconnection from the Yohkoh and SOHO satellites, especially in solar flares and coronal heating events. Much of it has been produced by Tsuneta and Shibata and their coworkers (e.g., Shibata et al., 1990; Kusano et al., 1995).

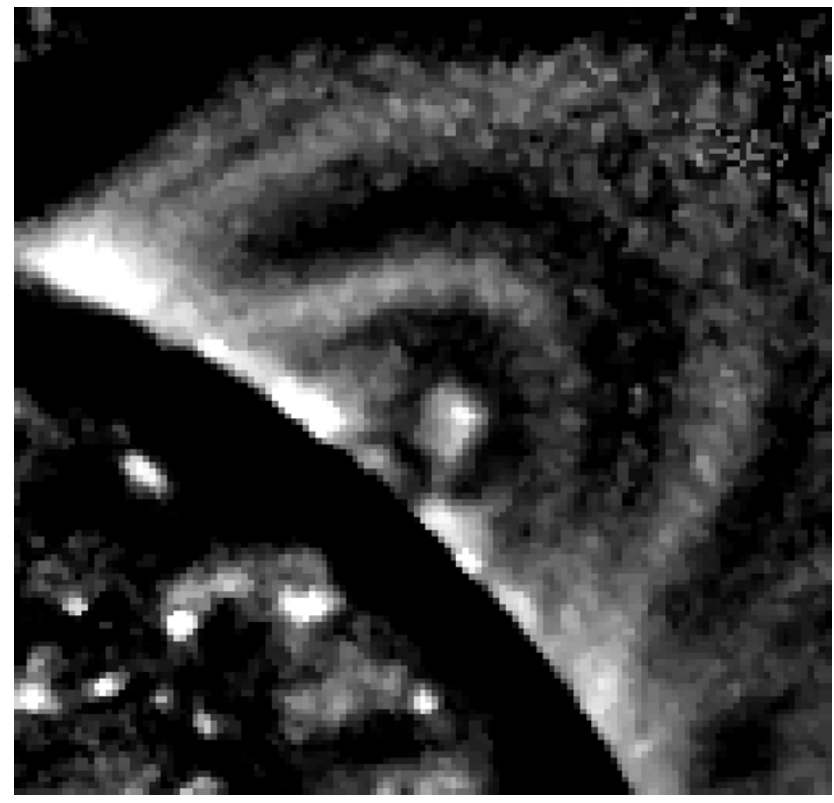

Fig. 12. Large-scale coronal loop whose heating may well be turbulent reconnection.

\subsection{Solar flares}

For example, after the large-scale magnetic field has been opened up in a coronal mass ejection, it closes back down by reconnection and produces below the reconnection point a rising cusp-shaped arcade that shows up in Yohkoh images. In a large eruptive solar flare, there is a similar magnetic process, where an arcade erupts because of a MHD catastrophe (Priest and Forbes, 1990; Amari et al., 2000) and then drives reconnection (Fig. 8). The reconnection-produced flare loops have a structure deduced from numerical experiments (Fig. 9) that agrees with many aspects of the observations. For example, the famous Tsuneta et al. (1992) flare has a set of rising soft X-ray loops (Fig. 10) with a temperature and pressure structure that agrees qualitatively with the models. Also, the equally famous Masuda et al. (1994) flare exhibits hard X-rays at the loop top and at its feet. Furthermore, McKenzie and Hudson (1999) have discovered above the flare loops a spikey current sheet (Fig. 11) containing dark voids moving down at $200 \mathrm{~km} / \mathrm{s}$ that may possibly be 

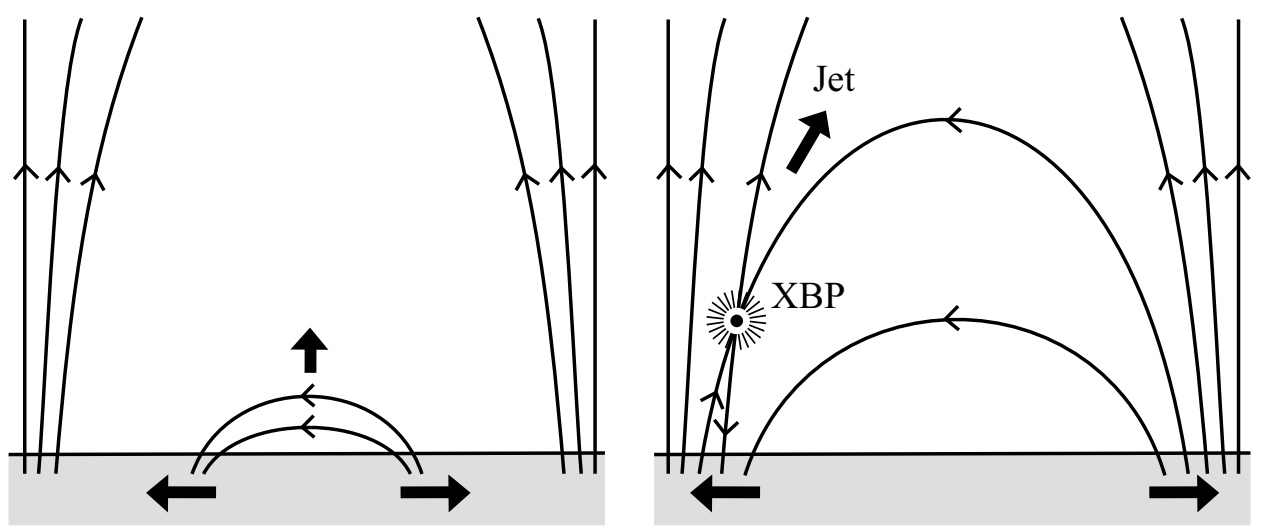

Fig. 13. Converging Flux Model.

produced by impulsive bursty reconnection (Priest, 1987).

\subsection{Coronal Heating}

Many examples of reconnection have been found in coronal heating events. The temperature profile measured along large-scale loops in Yohkoh images (Fig. 12), for instance, has been found to be consistent with heating by Parker braiding or turbulent reconnection (Priest et al., 1998, 2000b).

With Yohkoh too Yoshida and Tsuneta (1996) have shown that the hottest loops are either cusp-shaped or are interacting loops, both of which may be produced by reconnection. Furthermore, Shibata et al. (1996) has observed many x-ray jets produced by reconnection which have been simulated in a most impressive manner (Yokoyama and Shibata, 1997).

On SOHO the MDI instrument has shown that the surface of the Sun is covered with a magnetic carpet, in which magnetic fragments are continually emerging, fragmenting, merging and cancelling (Schrijver et al., 1997). Indeed, this is such an efficient process that the magnetic flux at the surface in the Quiet Sun is replaced not every 11 years, but every 40 hours.

The basic idea of the Converging Flux Model (Priest et $a l ., 1994)$ is that new flux emerges in a supergranule cell, moves to the boundary and reconnects (Fig. 13), where it can in large events create an X-ray bright point and accelerate an $\mathrm{X}$-ray jet. Furthermore, with the EIT instrument on SOHO, Falconer et al. (1998) have found that most bright points lie above oppositely directed magnetic fragments. With TRACE Parnell and Jupp (2000) have found that most nanoflares also lie above the supergranule boundaries.

So what is the effect of the magnetic carpet on coronal heating? I would like to suggest the following scenario. The evolution of magnetic flux on the solar surface has three stages. First of all, a bipolar ephemeral region is born once every few days in a supergranule cell of typical diameter $15 \mathrm{Mm}$ (Fig. 14(a)). This drives global reconnection with the overlying field which becomes visible as an X-ray bright point. Then each polarity of the ephemeral region moves towards the boundary over about 8 hours and fragments into typically 10 network elements (Fig. 14(b)). This drives internal reconnection between the network elements and also provides a fibril magnetic structure within which MHD waves driven by granulation may dissipate efficiently. Thirdly, over the next 22 hours, the network elements move

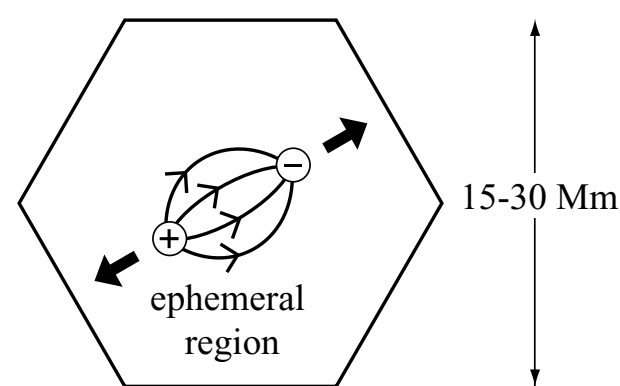

(a)

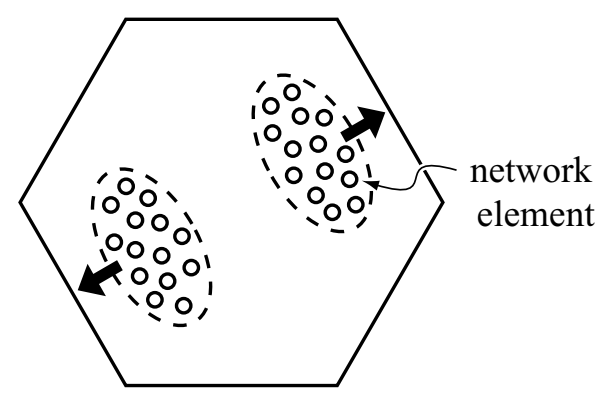

(b)

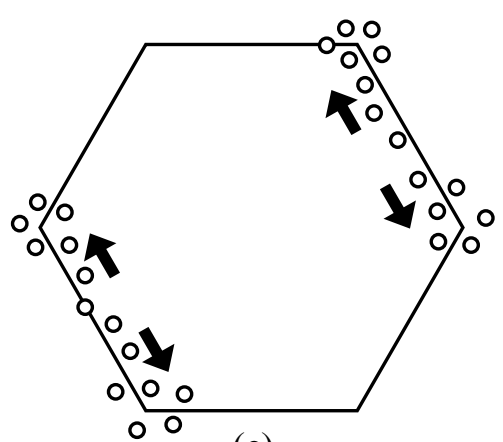

(c)

Fig. 14. The evolution of magnetic flux in the quiet Sun. 
along the boundary of the cell and undergo merging, fragmentation and cancellation (Fig. 14(c)), which also drive reconnection within the network and in the overlying field. The elementary heating events in this model are: binary reconnection between flux pairs, separator reconnection by the interaction of three or more sources, internal Parker braiding and dissipation of waves driven by granular buffeting.

\section{Concluding Comment}

In summary, there are two main points to this review: the theory of three-dimensional reconnection is developing rapidly; and SOHO and Yohkoh have produced much evidence of the consequences of reconnection. However, for a detailed understanding of the connection between the surface of the Sun and its corona, we eagerly await the Solar-B Mission.

Acknowledgments. I am most grateful to the Symposium organisers and the UK Particle Physics and Astronomy Research Council for financial support and to our Japanese hosts for the care with which they have organised a most stimulating meeting.

\section{References}

Amari, T., J. F. Luciani, Z. Mikic, and J. Linker, A twisted flux rope model for CME's and two-ribbon flares, Astrophys. J., 529, L49-L52, 2000.

Axford, W. I., Magnetic field reconnection, in Magnetic Reconnection in space and Laboratory Plasmas, edited by E. W. Hones, pp. 1-8, Amer. Geophys. Union, Washington, 1984.

Craig, I. J. D. and S. M. Henton, Exact solutions for steady-state incompressible magnetic reconnection, Astrophys. J., 450, 280-288, 1995.

Démoulin, P., J. C. Hénoux, E. R. Priest, and C. H. Mandrini, Quasiseparatrix layers in solar flares. I. Method, Astron. Astrophys., 308, 643655, 1996.

Falconer, D. A., R. L. Moore, and J. G. Porter, Micro-coronal bright points observed in the quiet magnetic network by SOHO/EIT, Proceedings of High-Resolution Solar Atmospheric Dynamics Workshop, 1998.

Galsgaard, K. and $\AA$. Nordlund, Heating and activity of the solar corona 3. Dynamics of a low-beta plasma with 3D null points, J. Geophys. Res., 102, 231-248, 1997

Galsgaard, K., C. E. Parnell, and J. Blaizot, Elementary heating events due to magnetic interactions between two flux surfaces, Astron. and Astrophys., 362, 395-405, 2000

Hesse, M. and K. Schindler, A theoretical foundation of general magnetic reconnection, J. Geophys. Res., 93, 5539-5567, 1988.

Hornig, G. and L. Rastätter, The magnetic structure of $B \neq 0$ reconnection, Physica Scripta, T74, 34-39, 1998.

Kusano, K., Y. Suzuki, and K. Nishikawa, A solar flare triggering mechanism based on the Woltjer-Taylor minimum energy principle, Astrophys. J., 441, 942-951, 1995.

Lau, Y.-T. and J. M. Finn, 3D kinematic reconnection in the presence of field nulls and closed field lines, Astrophys. J., 350, 672-691, 1990.

Longcope, D. W., Current sheet formation and reconnection of separator field lines, in Observational Plasma Physics: Five Years of Yohkoh and Beyond, edited by T. Watanabe, pp. 179-184, Kluwer Press, 1998.

Longcope, D. W. and S. C. Cowley, Current sheet formation along 3D magnetic separatices, Phys. Plasmas, 3, 2885-2897, 1996.

Longcope, D. W. and A. V. R. Silva, A current ribbon model for energy storage and release with application to the flare of 7 Jan. 1992, Solar Physics, 179, 349-377, 1998

McKenzie, D. E. and H. S. Hudson, X-ray observations of motions and structure above a solar flare arcade, Astrophys. J., 519, L93-L96, 1999.

Masuda, S., T. Kosugi, H. Hara, S. Tsuneta, and Y. Ogawara, A loop-top hard X-ray source in a compact solar flare, Nature, 371, 495-497, 1994.

Parnell, C. E. and P. E. Jupp, Statistical analysis of the energy distribution of nanoflares in the quiet Sun, Astrophys. J., 529, 554-569, 2000.

Parnell, C. E., J. Smith, T. Neukirch, and E. R. Priest, The structure of 3D magnetic neutral points, Phys. Plasmas, 3, 759-770, 1996.
Priest, E. R., Appearance and disappearance of magnetic flux at the solar surface, in Role of Fine-Scale Magnetic Fields on Structure of the Solar Atmosphere, edited by E. Schroter, M. Vazquez, and A. Wyller, pp. 297316, Camb. Univ. Press, 1987.

Priest, E. R. and P. Demoulin, 3D reconnection without null points, J. Geophys. Res., 100, 23,443-23,463, 1995

Priest, E. R. and T. G. Forbes, New models for fast steady-state magnetic reconnection, J. Geophys. Res., 91, 5579-5588, 1986.

Priest, E. R. and T. G. Forbes, Magnetic field evolution during prominence eruptions and two-ribbon flares, Solar Phys., 126, 319-350, 1990.

Priest, E. R. and T. G. Forbes, Magnetic Reconnection, Cambridge University Press, 2000

Priest, E. R. and L. C. Lee, Nonlinear reconnection models with separatrix jets, J. Plasma Phys., 44, 337-360, 1990.

Priest, E. R. and C. J. Schrijver, Aspects of 3D magnetic reconnection, Solar Phys., 190, 1-24, 1999.

Priest, E. R. and V. S. Titov, Magnetic reconnection at three-dimensional null points, Phil. Trans. Roy. Soc., 354, 2951-2992, 1996.

Priest, E. R., V. S. Titov, G. E. Vekstein, and G. J. Rickard, Steady linear X-point magnetic reconnection, J. Geophys. Res., 99, 21,467-21,480, 1994.

Priest, E. R., D. P. Lonie, and V. S. Titov, Bifurcations of magnetic topology by the creation or annihilation of null points, J. Plasma Phys., 56, 507530, 1996.

Priest, E. R., C. R. Foley, J. Heyvaerts, T. D. Arber, J. L. Culhane, and L. W. Acton, Nature of the heating mechanism for the diffuse solar corona, Nature, 393, 545-547, 1998.

Priest, E. R., V. S. Titov, R. E. Grundy, and A. W. Hood, Exact solutions for reconnective magnetic annihilation, Proc. Roy. Soc., 456, 1821-1849, 2000a.

Priest, E. R., C. R. Foley, J. Heyvaerts, T. D. Arber, J. L. Culhane, and L. W. Acton, A method to determine the heating mechanisms of the solar corona, Astrophys. J., 539, 1002-1022, 2000 b.

Sato, T., 3D reconnection between two colliding magnetized plasmas, Phys. Rev. Lett., 54, 1502-1505, 1985.

Schindler, K., M. Hesse, and J. Birn, General magnetic reconnection, parallel electric fields and helicity, J. Geophys. Res., 93, 15,477-15,485, 1988.

Schrijver, C. J., A. M. Title, K. L. Harvey, N. R. Sheeley, Y.-M. Wang, G. H. J. van den Oord, R. A. Shine, T. D. Tarbell, and N. E. Hurlburt, Largescale coronal heating by the dynamic, small-scale magnetic field of the Sun, Nature, 48, 424-425, 1997.

Shibata, K., S. Nozawa, R. Matsumoto, A. C. Sterling, and T. Tajima, Emergence of solar magnetic flux from the convection zone into the photosphere and chromosphere, Astrophys. J., 351, L25-L28, 1990.

Shibata, K., M. Shimojo, T. Yohoyama, and M. Ohyama, Theory and observations of $\mathrm{x}$-ray jets, in Magnetic Reconnection in the Solar Atmosphere, edited by R. D. Bentley and J. T. Mariska, pp. 29-38, 1996.

Sonnerup, B. U. O. and E. R. Priest, Resistive MHD stagnation-point flows at a current sheet, J. Plasma Phys., 14, 283-294, 1975.

Strachan, N. R. and E. R. Priest, A general family of non-uniform reconnection models with separatrix jets, Geophys. Astrophys. Fluid Dynamics, 74, 245-274, 1994.

Titov, V. S., P. Dèmoulin, and G. Hornig, Quasi-separatrix layers: refined theory and its application to solar flares, Proc. of The 9th European Meeting on Solar Physics Magnetic Fields and Solar Processes, Florence, Italy, ESA SP-448, 715-722, 1999.

Tsuneta, S., H. Hara, T. Shimizu, L. W. Acton, K. T. Strong, H. S. Hudson, and Y. Ogawara, Observation of a solar flare at the limb with the Yohkoh Soft X-ray Telescope, Publ. Astron. Soc. Japan, 44, L63-L69, 715-722, 1992.

Ugai, M. and W. B. Wang, Computer simulations on 3D plasmoid dynamics by spontaneous fast reconnection, J. Geophys. Res., 103, 4573-4585, 1998.

Yokoyama, T. and K. Shibata, Magnetic reconnection coupled with heat conduction, Astrophys. J., 474, L61-L64, 1997.

Yoshida, T. and S. Tsuneta, Temperature structure of solar active regions, Astrophys. J., 459, 342-351, 1996.

E. R. Priest (e-mail: eric@mcs.st-and.ac.uk) 